\title{
Flow characteristics of nerve block Y-connectors
}

\section{T. Al-Ani, University Hospital Hairmyres, Glasgow, East Kilbride, UK}

\section{Background and Aims:}

The use of nerve block Y-connectors is common in regional anaesthesia practice. We usually attach an elastomeric pump to the Y-connector (YC) in order to deliver two streams of local anaesthetic (LA). Do these YCs deliver equal volumes of LA at all times?

\section{Methods:}

The volumes ( $\mathrm{ml})$ of the LA infused at both ends of the Pajunk YC were calculated after:

(1) An elastomeric-pump infusion attached to the $\mathrm{YC}$, with no external resistance applied to flow to either limbs of the YC.

(2) An elastomeric-pump infusion attached to the $\mathrm{YC}$, with external resistance to flow applied to either limbs of the YC.

(3) A $20 \mathrm{ml}$ syringe injection attached to the YC, with no external resistance to flow applied to either limbs of the YC.

(4) A $20 \mathrm{ml}$ syringe injection attached to the $\mathrm{YC}$, with external resistance to flow applied to either limbs of the YC.

All repeated 15 times.

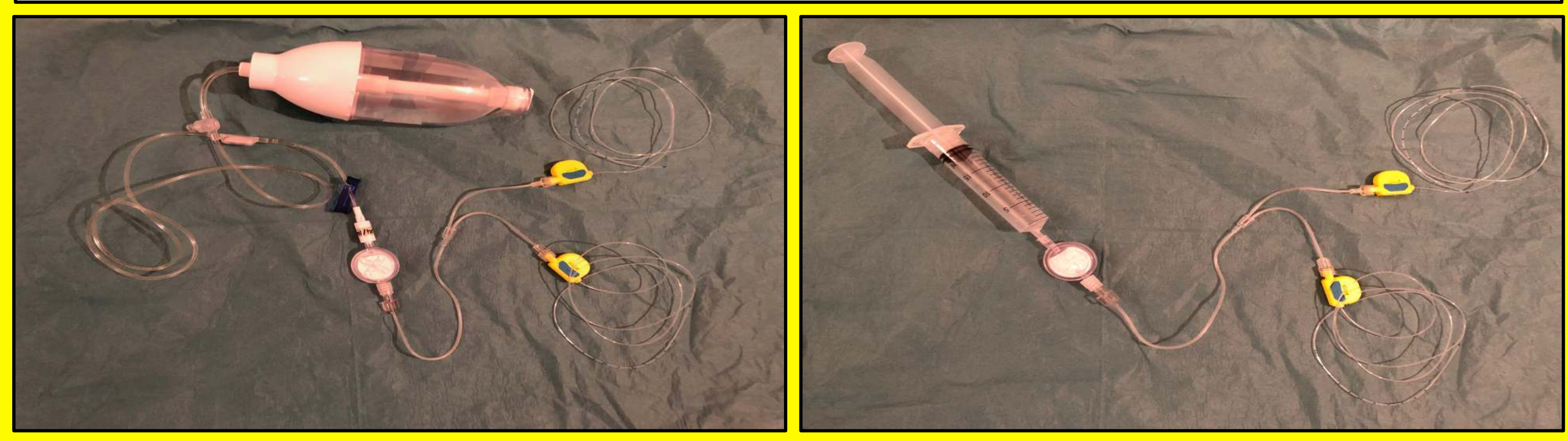

\begin{tabular}{|c|c|c|c|c|c|c|c|}
\hline \multicolumn{8}{|c|}{ Results: } \\
\hline \multicolumn{2}{|c|}{$\begin{array}{l}\text { Elastomeric pump: } 300 \mathrm{ml} \text { at } \\
8 \mathrm{ml} / \mathrm{hour} \text { (with no resistance } \\
\text { to flow applied to either limbs } \\
\text { of the YC) } n=15\end{array}$} & \multicolumn{2}{|c|}{$\begin{array}{l}\text { Elastomeric pump: } 300 \mathrm{ml} \text { at } \\
8 \mathrm{ml} / \text { hour (with resistance to } \\
\text { flow applied to either limbs of } \\
\text { the YC) } n=15\end{array}$} & \multicolumn{2}{|c|}{$\begin{array}{l}20 \mathrm{ml} \text { injection (with no } \\
\text { resistance to flow applied to } \\
\text { either limbs of the YC) } \\
n=15\end{array}$} & \multicolumn{2}{|c|}{$\begin{array}{l}20 \mathrm{ml} \text { injection (with resistance } \\
\text { to flow applied to either limbs } \\
\text { of the YC) } \\
n=15\end{array}$} \\
\hline $\begin{array}{l}\text { YC right limb } \\
\text { (ml) }\end{array}$ & $\begin{array}{l}\text { YC left limb } \\
\text { (ml) }\end{array}$ & $\begin{array}{l}\text { YC limb with } \\
\text { resistance (ml) }\end{array}$ & $\begin{array}{l}\text { YC limb with no } \\
\text { resistance (ml) }\end{array}$ & $\begin{array}{l}\text { YC right limb } \\
\text { (ml) }\end{array}$ & $\begin{array}{l}\text { YC left limb } \\
\text { (ml) }\end{array}$ & $\begin{array}{l}\text { YC limb with } \\
\text { resistance (ml) }\end{array}$ & $\begin{array}{l}\text { YC limb with no } \\
\text { resistance (ml) }\end{array}$ \\
\hline 125.3 & 174.7 & 98.1 & 201.9 & 9.5 & 10.5 & 7.8 & 12.2 \\
\hline 163 & 137 & 104.1 & 195.9 & 10.6 & 9.4 & 7.5 & 12.5 \\
\hline 156.2 & 143.8 & 100.5 & 199.5 & 9 & 11 & 8 & 12 \\
\hline 135 & 165 & 97.2 & 202.8 & 9.7 & 10.3 & 6.9 & 13.1 \\
\hline 129.5 & 170.5 & 93 & 207 & 8.7 & 11.3 & 7.4 & 12.6 \\
\hline 162 & 138 & 109.1 & 190.9 & 12 & 8 & 7 & 13 \\
\hline 154.1 & 145.9 & 98.3 & 201.7 & 9.5 & 10.5 & 6.9 & 13.1 \\
\hline 136.7 & 163.3 & 91.5 & 208.5 & 11 & 9 & 7.8 & 12.2 \\
\hline 162 & 138 & 89.9 & 210.1 & 10.2 & 9.8 & 7.9 & 12.1 \\
\hline 158.2 & 141.8 & 81.6 & 218.4 & 10.9 & 9.1 & 6.8 & 13.2 \\
\hline 108.1 & 191.9 & 102.7 & 197.3 & 9 & 11 & 7.1 & 12.9 \\
\hline 144.3 & 155.7 & 94 & 206 & 9.5 & 10.5 & 7.7 & 12.3 \\
\hline 140.7 & 159.3 & 90.5 & 209.5 & 10.6 & 9.4 & 8 & 12 \\
\hline 170.1 & 129.9 & 95.9 & 204.1 & 10.3 & 9.7 & 7.3 & 12.7 \\
\hline 137 & 161 & 88.3 & 211.7 & 9 & 11 & 7.9 & 12.1 \\
\hline
\end{tabular}

\section{Conclusions:}

